\title{
La enseñanza de la historia y las ciencias sociales hoy: contrasentidos y posibilidades ${ }^{1}$
}

\author{
Teaching History and Social Sciences Today: \\ Contradictions and Possibilities
}

\author{
O ensino da história e as ciências sociais na atualidade: \\ contra-sentidos e possibilidades
}

Alcira Aguilera Morales²

\section{Resumen}

El presente artículo revisa críticamente algunos lugares y supuestos que damos por ciertos en el campo de la enseñanza de la historia y las ciencias sociales en Colombia, y que quizá no dejan problematizar y buscar alternativas en la enseñanza de estas disciplinas. Para ello interesó revisar varios documentos balance que se han escrito sobre el asunto y desde allí reconstruir contrasentidos, tensiones, lugares comunes y los retos que constituyen para el campo.

\section{Palabras clave}

enseñanza de la historia; integración curricular; interdisciplinariedad escolar; ciencias sociales

\section{Abstract}

This paper presents a critical review of some ideas and assumptions we take as a given in the field of teaching History and Social Studies in Colombia, and which might prevent problematizing and looking for alternatives in the teaching of those disciplines. For this purpose, we reviewed a number of balance documents on the topic, and re-constructed the contradictions, tensions, commonplaces and challenges that make up the field.

\section{Keywords}

teaching of History; curricular integration; school cross-curricularity; social studies

\section{Resumo}

0 presente artigo revisa criticamente alguns lugares e premissas que acreditamos verdadeiras no campo do ensino da história e as ciências sociais na Colômbia, e que tal vez não permitem problematizar e procurar alternativas no ensino dessas disciplinas. Para isso, foram revisados vários documentos balanço, escritos sobre o tema, e desde estes reconstruir contra-sentidos, tensões, lugares comuns e os retos que constituem para o campo.

\section{Palavras chave}

ensino da história; integração curricular; interdisciplinaridade escolar; ciências sociais

Artículo recibido el 16 de mayo del 2016 y aprobado el 21 de diciembre del 2016

1 El presente artículo hace parte de la investigación financiada por el Centro de Investigaciones de la Universidad Pedagógica (ciup), titulada "Estado del arte sobre la enseñanza de la historia y las ciencias sociales en las facultades de Humanidades y Educación de la upN (2000-2014)" durante el 2015. El proyecto contó con la participación de los profesores María Isabel González y Alfonso Torres, y estuvo coordinado por la suscrita.

2 Profesora Investigadora de la Universidad Pedagógica Nacional, grupo de investigación Sujetos y Nuevas Narrativas en la Investigación y la Enseñanza de las Ciencias Sociales, de la Universidad Pedagógica Nacional. Correo electrónico: aamorales@pedagogica.edu.co 
$\mathrm{Al}$ analizar los balances que se han realizado sobre la enseñanza de la historia y las ciencias sociales, en lo corrido del siglo XXI, encontramos que, por un lado, se ha naturalizado la idea de que se ha abandonado la enseñanza de historia en la escuela por la llamada integración de las ciencias y las ciencias sociales. En segundo lugar, el problema de qué se enseña ha sido desplazado por el control evaluativo, lo cual propicia el abandono de la discusión curricular, y del sentido de los contenidos históricos y sociales en la enseñanza. Con estas primeras intuiciones se interrogó en los balances por el lugar de la enseñanza de la historia en las ciencias sociales, los asuntos que permanecen y los avances que se enuncian a partir de estos.

\section{Entre contrasentidos}

\section{Entre ley y prácticas el trecho es abismal}

Quisiera plantear los hallazgos iniciales, en términos de contrasentidos, entendiendo por ellos algunos enunciados que se han naturalizado en nuestra cultura académica pero que en algunos discursos y prácticas evidencian lo contrario. Un primer contrasentido se ha instaurado al asumir que lo que dictaminan las políticas públicas regula lo que se enseña en historia y ciencias sociales. Al respecto, podemos mencionar la exigencia legislativa respecto a la integración de las ciencias sociales, acompañada del fracaso integracionista.

Si bien debería existir una relación directa entre lo que la ley establece y lo que se enseña en historia, encontramos divergencias en esta relación. Esto se sustenta en que, en términos legislativos, se suele aludir, de manera taxativa, a la integración de las ciencias sociales a partir de la reforma curricular de 1984, que a través del Decreto 1002 establecía la integración de la historia y la geografía bajo la denominación del "área de ciencias sociales", con lo cual buscaba integrar las dos disciplinas por tradición enseñadas con otras propias de las ciencias sociales ${ }^{3}$

3 Durante gran parte del siglo $x x$, se hacía énfasis en la enseñanza de la historia y la geografía separadas, aunque en la realidad la segunda se volvió un apéndice de la primera.
(Guerrero, 2011; Restrepo, Ramos Reyes y Sarmiento Lozano, 2000; Rodríguez, 2014). De esta manera, historia, geografía, y educación para la democracia, la paz y la vida social fueron agregados que constituyeron la llamada área de ciencias sociales. Sin embargo, los cuestionamientos a la integración y a su incidencia en la enseñanza se dieron desde las posturas que consideraban que la integración invisibilizó el papel de la historia en la formación de las nuevas generaciones (Guerrero, 2011), hasta quienes veían en la integración una propuesta innovadora que superaría la enseñanza tradicional, episódica y memorística de la historia, de allí que se llegara a considerar que

El Decreto 1002 de 1984 se explica por el agotamiento de la concepción tradicional de enseñanza, sumado a que se retoman aportes de científicos sociales del mundo universitario, criterios metodológicos de la pedagogía activa y planteamientos de la psicología cognitiva, para así hacer frente a la tecnología educativa. (Torres Carrillo, 1995).

Pero aunque la integración corría por cuenta de la legislación educativa, en materia de formación docente, de textos escolares y en las mismas prácticas docentes la asignaturización era evidente. Por eso, quienes cursaron la primaria o el bachillerato a finales de la década de los ochenta se encontraban con una práctica común en la que

la historia y la geografía eran asignaturas de las cuales se obtenían calificaciones independientes, que debían ser computadas con una nota adicional que se ordenaba de la materia de democracia (educación para la democracia, la paz y la vida social) y el promedio correspondía al área de ciencias sociales. (Guerrero, 2011).

Así, bajo la denominación del área de ciencias sociales lo que se integraba era el promedio de las tres grandes asignaturas que la constituían.

En torno a la edición de los libros de texto también se refleja la fragmentación, tal como lo expresa el equipo de docentes que lideraba la Maestría en Enseñanza de la Historia de la Universidad Pedagógica Nacional (UPN): 
[...] al revisar los textos escolares se encuentra que las nuevas orientaciones no alcanzaron mayores efectos en la enseñanza [...]. La integración de estas dos disciplinas se hizo intercalando temas históricos y geográficos, dejando a un lado los aportes de otras disciplinas, como la antropología, la sociología, la etnografía, etc., en el tratamiento de los temas. (Equipo de Maestría en Enseñanza de la Historia del Departamento de Ciencias Sociales, UPN, 1999, p. 9).

Este panorama no variaría mucho en la década de los noventa ${ }^{4}$, recordemos que en este periodo se emprende una serie de contrarreformas educativas ${ }^{5}$. En este escenario, la Ley General de Educación, Ley 115 de 1994, también apostaría a la integración de las disciplinas (art. 21). Además de ello, se señalaba que "los currículos debían formularse con arreglo a los indicadores de logro establecidos por el MEN" (Medina, 2008, p. 17).

Lo que se encuentra desde entonces es que ni las empresas editoriales, ni la formación docente comprendían el llamado a la integración. De allí que no fuese cierto que desapareció la enseñanza de la historia, pues esta disciplina se mantuvo pese a la etiqueta integracionista. De acuerdo con Guerrero (2011), la Ley General de Educación no logró construir un asidero epistemológico para eso que se denomina "ciencias sociales", a la vez que se establecen distancias entre la historia y la geografía, y la pedagogía y la didáctica para lograr avanzar en la enseñanza del área. Estos problemas en el campo se amplían con la dispersión generada por la creación de nuevos accesorios para el área, como las cátedras en ética y valores, constitución política y democracia, estudios afrocolombianos, derechos

4 En 1989, se decretó la enseñanza de la historia, geografía y cívica, a través del Decreto 1167, pero este no cambiaba la lógica mencionada anteriormente.

5 Según Martinic (2001), son reformas de segunda generación, las cuales se orientan a la calidad y la equidad, es decir se asume un ideal de calidad ligado al progreso pero también a una lógica evaluativa eficientista en la que se correlacionan recursos con resultados. Las de primera generación las ubica en la década de los ochenta y responde a la ampliación de la cobertura educativa, la descentralización acompañada de la reducción del gasto educativo. Las de tercera generación responden a las políticas actuales, profundizan en una perspectiva de autonomía de las escuelas y la descentralización. humanos (DDHH) y más recientemente la anunciada Cátedra para la Paz.

En los primeros lustros del siglo xxi, con la emisión de los "Lineamientos curriculares de ciencias sociales" - política emanada en el 2002-, desde una perspectiva multidisciplinar, que se sostiene en el trabajo pedagógico en torno a ejes generadores, desde un enfoque curricular abierto, integrado, en espiral y flexible, que cuestiona la primacía disciplinar $^{6}$, se intentaría mantener la mirada integracionista del área. Pero al analizar con detenimiento hasta dónde se ha integrado, reaparecen los viejos problemas.

En primer lugar, en un trabajo de Medina (2008) para el Convenio Andrés Bello ${ }^{7}$, se presenta una revisión de los textos escolares en ciencias sociales para la educación básica y media en Colombia. En resumen, el autor encuentra que en los textos de la editorial Educar se da especial atención a la construcción de ciudadanía, la educación cívica, la formación en valores y los derechos humanos. En el caso de la serie Santillana, hay una importante dedicación a la formación en los conocimientos disciplinares de la historia, la geografía, la economía y la sociología. En ellos la historia no pierde su especificidad, y se reconocen claramente los límites disciplinares entre unas y otras ciencias sociales. En el caso de las series publicadas por el

6 No es el interés en este documento mencionar las críticas y debates que suscitó el documento "Lineamientos curriculares", para ello sugerimos revisar los análisis de Medina (2008) sobre la mirada ahistórica y presentista que lo constituye. También Guerrero (2011) presenta la confusión epistemológica y formativa que implica hablar de ciencias sociales interdisciplinares. De igual manera el cuestionamiento a lo interdisciplinar se ve en las pruebas de Estado que, en manos del Icfes desde el 2000, también dividían las ciencias sociales en historia, geografía y filosofía, principalmente (Ortiz, Ayala Diago, Chaparro, Sarmiento Lozano y Restrepo, 2007).

7 Este trabajo busca contribuir al desarrollo de una cultura de la paz, la integración y la cooperación de los pueblos de la Comunidad Andina, y aborda un análisis de la legislación, los textos escolares y los contenidos curriculares que aporten a esta mirada integracionista. En este trabajo se escogieron las siguientes series de textos de ciencias sociales: Ciencias sociales, propuesta curricular integrada, serie publicada en el 2003 por Educar; Identidades, publicada por Norma en el 2004; Nuevo Identidades, publicada también por Norma en el 2005; y la serie de Santillana, una para básica primaria bajo el título de Amigos de las ciencias sociales publicada en el 2006, y otra para básica secundaria: Contextos sociales, publicada en el 2004. 
Grupo Editorial Norma, Medina (2008) menciona que se reconoce el trabajo en valores, derechos y formación ciudadana, a la vez que la historia se percibe bien delimitada en los contenidos, al igual que la geografía, la economía y la sociología.

En estas miradas de la historia que se enseña prevalece el abordaje de una disciplina cronológica con los periodos históricos de otrora. En el caso de la geografía, se omite su dimensión latinoamericana y se relaciona con la ubicación de Colombia en relación con las fronteras y países vecinos. Este análisis de los textos editados en el presente siglo sigue dando vigencia a la idea de que se ha mantenido la enseñanza de la historia y la geografía, con algo de economía y cívica. Esta postura es complementada por Arias (2015), quien encuentra que lo que los estudiantes recién egresados de la educación media evocan de sus clases de ciencias sociales se refiere principalmente a contenidos históricos como fechas insignes, personajes históricos, acontecimientos políticos y militares relevantes, y recortes de periodos históricos instaurados por esta disciplina, entre otros. Esta conclusión se complementa con los contenidos disciplinares que encuentra en los libros de texto actuales, en los que se menciona un amplio temario de contenidos históricos.

\section{Formar disciplinarmente para pensar interdisciplinarmente}

Ello nos lleva a ubicar un contrasentido en las posibilidades que se han instaurado sobre la interdisciplinariedad que convoca a las ciencias sociales versus la disciplinariedad que prima en la enseñanza de estas (Aguilera y González, 2009). Esto se hace a partir de qué hablar de interdisciplinariedad o multidisciplinariedad en las ciencias sociales escolares cuando no contamos con estructuras académicoinvestigativas que incidan en la formación pedagógica y en la enseñanza interdisciplinaria de estas.

Esto lo podemos ilustrar acudiendo a los sentidos que orientan la formación de licenciados en el campo. Al respecto Rodríguez (2013) presenta un rastreo de los programas de formación de docentes de ciencias sociales que existen en Colombia posteriores al Decreto 272 de 1998 (el cual reglamenta la titulación, principios de funcionamiento de los programas ofrecidos por las universidades, entre otros aspectos). Allí menciona que la mayoría de programas que se ofrecen se nomina "Licenciatura en Educación Básica con Énfasis en Ciencias Sociales (Lebecs)", solo la Universidad del Valle ofrece dos licenciaturas en Historia. En la actualidad se encuentran activos 29 programas:

Todos los programas cuentan con espacios académicos de fundamentación epistemológica e investigativa en ciencias sociales [...] En cuanto al lugar que ocupa la historia en la formación específica se encuentran tres énfasis en la formulación de los planes de estudio: en un importante número de programas prevalece la formación en historia y geografía en ordenaciones cronológicas (historia universal, historia antigua e historia moderna) y en ramas y conceptos de la geografía (geografía física, geografía humana, climatología, geomorfología). En un grupo menor de programas prevalece la formación interdisciplinar, asociada a la agrupación temática por categorías (espacio-tiempo), conceptos (instituciones, territorio, ambiente, sociedad, poder y cultura) y por procesos y problemas (socialización y cambio social) como ocurre en la Lebecs de la Universidad Distrital. (Rodríguez, 2013, p. 43).

Si en la mayoría de programas de formación para los futuros docentes de ciencias sociales prima la formación disciplinar, ¿cómo hacer los desplazamientos para una enseñanza interdisciplinar? Es decir, ¿cómo, desde los programas de formación, se dota de contenido esa nominación interdisciplinar? Pues lo que se constata es la propia fragmentación disciplinar en las universidades, y la ausencia de investigaciones interdisciplinares (Restrepo et al., 2000) que estén relacionadas con la educación, la pedagogía y los campos disciplinares que constituyen las ciencias sociales.

Lo que evidencia este contrasentido es que no se trata de una disputa entre disciplinariedad e interdisciplinariedad, sino de formarse disciplinarmente para pensar y actuar interdisciplinarmente a la hora de enseñar y abordar investigativa y pedagógicamente las ciencias sociales, teniendo claras las diferencias en los desarrollos interdisciplinares desde las 
ciencias sociales y las ciencias sociales escolares. Al respecto, las experiencias de grupos y escuelas que tienen este carácter interdisciplinar evidencian que la formación disciplinar mediada por los contextos epocales de los diferentes actores posibilitó luego desarrollar propuestas interdisciplinarias ${ }^{8}$.

Lo anterior implica que los retos epistemológicos y metodológicos propios de las relaciones interdisciplinares se asumen acudiendo a las disciplinas. Por ejemplo, Restrepo et al. (2000) ubican en la historia la disciplina capaz de hacer tránsitos interdisciplinares:

Dado que la historia moviliza los saberes de todos los ámbitos, dicha disciplina se convierte en el eje pedagógico por excelencia para una formación integral en ciencias sociales, siempre que los/as maestros/as posean la ductilidad suficiente para integrar en la exposición temporal las distintas dimensiones analíticas e históricas de los distintos ámbitos de las ciencias sociales. (p. 22).

En esta misma perspectiva, el profesor Mauricio Archila (2004), al preguntarse si se enseña historia o ciencias sociales, encuentra en la historia el lugar en el que convergen no solo las otras disciplinas, sino también métodos y lenguajes de otras ciencias sociales. Así, para él, la historia es el eje interdisciplinar desde el cuál también se incorporan teorías de las demás ciencias sociales. Es decir, para lograr realizar tránsitos interdisciplinares es indispensable la formación disciplinar, pues es desde la disciplina - en este caso de la historia- que se pueden construir puentes que pongan en diálogo la enseñanza de las ciencias sociales.

8 Por ejemplo los grupos que tienen apuestas interdisciplinares, aunque se definen como una red multidisciplinar, como el de "Modernidad y colonialidad" tiene entre sus especialistas a sociólogos (Aníbal Quijano, Edgardo Lander, Ramón Grosfoguel), semiólogos (Walter Mignolo, Zulma Palermo), pedagogos (Caherine Walsh), antropólogos (Arturo escobar, Fernando Coronil), filósofos (Enrique Dussel, Santiago Castro Gómez, María Lugones); la Escuela de Birmingham, que recoge tradiciones marxistas y culturalistas, para constituir la teoría de los estudios culturales, contó con los aportes de Richard Hoggart (sociólogo) y Raymond Williams (sociólogo), Edward Palmer Thompson (historiador), Stuart Hall (sociólogo, teórico cultural). Estas apuestas intelectuales se expresan en una sólida formación disciplinar, que luego en el trabajo con otras disciplinas posibilitó el diálogo interdisciplinar.
Esta segmentación disciplinar también se constata en el ámbito evaluativo. Por lo menos en el caso de las pruebas de Estado, adelantadas por el Icfes, Ortiz et al. (2007) encuentran que desde el 2000 la prueba incorpora aspectos de otras ciencias sociales como economía, sociología, ciencia política y antropología, aunque "bajo el paraguas disciplinar de la historia y la geografía” (p. 17).

Los retos se ponen más difíciles cuando se abordan propuestas epistémicas que reconsideran las relaciones disciplinares instituidas por los desarrollos de la ciencia moderna. Este no es asunto que nos concierna en este momento, pero vale la pena mencionar cómo el reto hoy no es ni siquiera integrar las ciencias sociales sino volver a rehacer la relación entre la humanidad y la naturaleza, el corazón y el pensar, formas epistémicas que nos posicionan de una manera distinta frente a la realidad y a las formas de pensar que se convierten en acción creadora y transformadora de estas (De Sousa, 2009). Este reto pone la enseñanza de las ciencias sociales en la búsqueda de metodologías, de contenidos y alternativas de pensamiento/conocimiento (epistémicos) que orienten el sentido de estas en los escenarios educativos.

\section{Entre las disciplinas escolares o las disciplinas sociales}

Un tercer contrasentido que se evidencia en esta revisión tiene que ver con la idea de que existe una distancia abismal entre lo que se produce desde las disciplinas científicas y lo que se enseña, es decir entre la historia del historiador y la historia que se enseña. Evidentemente no todo lo que producen las disciplinas sociales es objeto de su enseñanza, puesto que el filtro final lo realizan los docentes desde sus diferentes posturas epistémicas. Para ilustrar este contrasentido partimos de revisar las visiones que sobre ciencias sociales se establecen en los balances y la manera como se relacionan con los conocimientos escolares.

En algunos trabajos se alude al origen de las ciencias sociales como disciplinas científicas, $y$ se acude al trabajo de la comisión Gulbenkian, coordinada por Immanuel Wallerstein (Aguilera 
y González, 2009; Álvarez, 2007; Ortiz et al., 2007; Restrepo et al., 2000). En este trabajo se recogen tanto las discusiones epistemológicas que dieron origen a las llamadas ciencias sociales desde una mirada eurocéntrica, como los retos que enfrentan hoy (Wallerstein et al., 1996; Wallerstein, 1997). En este análisis las disciplinas científicas anteceden las preguntas por su enseñanza. Esta es una mirada que pone de manifiesto la importancia del conocimiento epistemológico de las ciencias sociales como aspecto que estructura y define las rutas de enseñanza de la historia, la geografía y las demás ciencias sociales.

Sin embargo, en una revisión genealógica se muestra que el vínculo entre enseñanza de las ciencias sociales y la configuración de las disciplinas sociales fue muy estrecho a principios del siglo xx, e incluso que fue la enseñanza de las ciencias sociales la que permitió la posterior configuración de las disciplinas enseñadas. Es decir que los antecedentes de las ciencias sociales modernas en Colombia fueron las ciencias sociales escolares, acontecimiento ubicado entre 1930-19609 (Álvarez, 2007). El autor rastrea la manera como se erigen los conocimientos escolares asociados a las ciencias sociales:

El nombre que se le dio a este conjunto de saberes que comenzaron a proponerse para ser enseñados en la escuela fue el de estudios sociales. No hay referencia en ambientes diferentes de la existencia de los estudios sociales, como un todo, tal como se estructuraron en la escuela sobre los ejes del pasado, el territorio y la sangre. Durante los años treinta y cuarenta se hablaba en ocasiones de Ciencias Sociales y de manera más generalizada de estudios sociales. (Álvarez, 2007, p. 285).

Según ello, fue bajo la etiqueta de estudios sociales que se dio la invención de una historia, una geografía y una antropogeografía destinadas a consolidar un ideario nacional. En el caso de la antropogeografía, este saber entró a ser un asunto

9 Esta tesis sustenta que no existe total dependencia de las disciplinas escolares de las disciplinas académicas, sino que en algunos casos las disciplinas escolares preceden cronológicamente a sus disciplinas madre, así se presenta en el caso brasilero, colombiano, francés y español (Álvarez, 2007). estratégico para la identidad nacional y la administración del pueblo, pues desde ella se erigió la pretendida identidad nacional y se recuperó el papel de los pueblos indígenas colombianos (Álvarez, 2013) ${ }^{10}$. Este origen de las ciencias sociales cuestiona el tan nombrado distanciamiento entre las disciplinas y su enseñanza.

En las últimas tres décadas hemos asistido a una recuperación de dicho vínculo, que aunque no es generalizable, sí resulta potente, pues evidencia cómo la producción en los campos disciplinares, especialmente en la historia, sí ha tenido relaciones con los desarrollos pedagógicos y didácticos de su enseñanza.

En la década de los setenta, los desarrollos de la Nueva historia, centrados en la crítica a las corrientes positivistas instauradas por la historiografía tradicional que se encargó de mantener y consolidar una visión oficial de la historia, se constituyó en un referente importante que tendría incidencia en la enseñanza de la historia. Los ecos de esta corriente historiográfica en los años ochenta se encuentran en que,

En los textos escolares de historia de los ochenta se enfrentaron dos enfoques historiográficos contradictorios: el de historia episódica, anecdótica, descriptiva, nacionalista y romántica encarnada por algunos miembros de la Academia Colombiana de Historia y los enfoques socioeconómicos, estructurales y explicativos de la llamada Nueva Historia, representada por los autores de los textos escolares polémicos Rodolfo de Roux (1985) y Salomón Kalmanovitz (1989). (Equipo de Maestría, UPN, 1999, p. 9).

A su vez, en la década de los noventa tuvieron un eco importante los trabajos Historia oral ${ }^{11}$ e

10 Según Álvarez (2007), este nexo entre pedagogía y disciplinas escolares se debilitó debido a la influencia de las ideas del desarrollo del capitalismo transnacional, en aras de consolidar campos epistemológicos especializados, asunto que se ubica en la década de los sesenta, momento en que se institucionalizan las ciencias sociales en las universidades colombianas.

11 En esta tendencia se encuentran varios de los trabajos realizados en el marco de la Maestría en Enseñanza de la Historia de la UPN, que tuvo una única promoción (1998-2000). De igual manera se puede recoger los trabajos de los profesores Gina Velasco y Ricardo Castaño (2006), Renán Vega y Ricardo Castaño (1999), Renán Vega (1999), y Fabio Castro (2004). 
Historia desde abajo, en los que se cuestionan las miradas elitistas y excluyentes de la historia patria tradicional (Archila, 2004; Méndez Montenegro, 2002). Desde esta perspectiva, los trabajos de Vega (1998), y el "Proyecto de Investigación Histórica Escolar" adelantado por el profesor Castro Bueno (2004) evidencian las propias experiencias docentes que vinculan estas perspectivas históricas, en las que las metodologías de investigación social y la confrontación de la historia tradicional se reevalúan para dar un lugar distinto a los sujetos de la historia.

De igual manera, dentro de esta perspectiva se cuestionan los descuidos en la enseñanza de la historia al seguir acudiendo a los mismos mitos e hitos, a partir de nuevos aportes basados en la categoría "experiencia" 12 de Edward Palmer Thompson, ya que desde ella se busca explicar y explicarse el mundo cambiante. Desde esta perspectiva no existe "una historia para investigadores y estudiosos y otro tipo o 'historiecita' para licenciados y maestros" (Betancourt, 2008). De acuerdo con el autor, se trata de establecer una propuesta de enseñanza de la historia a tres niveles: nivel historiográfico (comprensión de la categoría analítica desde las tendencias y escuelas historiográficas); los hechos históricos en el programa (delimitar hechos concretos después de la revisión historiográfica, en un diseño conjunto con los estudiantes-maestros, selección que parte de la experiencia vivida y percibida por estos actores); $y$ la crítica a los textos (mostrar las inconsistencias presentadas en textos y manuales escolares sobre los temas abordados).

Son evidentes los diálogos entre disciplinas científicas y su enseñanza, y muestra de ello se rastrea en los trabajos de Las rutas pedagógicas del IDEP ${ }^{13}$, en los que se pone de manifiesto las propuestas de enseñanza de la historia desde la formación en el pensamiento crítico, en el entendido de que

12 En palabras de Thompson (1987), la experiencia expresa las relaciones con las que el sujeto experimenta las condiciones materiales de existencia, que se amplían a las relaciones culturales, de valor, afectivas, religiosas y sociales en general, pero a la vez que las experimenta, las reproduce o las transforma por medio de sus prácticas.

13 Desde el 2000 el IDEP ha venido desarrollando Las rutas pedagógicas en la enseñanza de la historia. Ampliar en Cortes et al. (2011). el maestro interroga o interpela críticamente el conocimiento, la evaluación, el currículo, las relaciones de convivencia, etc., y es precisamente a partir de todo este conjunto que termina formulando alternativas de enseñanza así estas no cuenten siempre con registros sistemáticos. (Cortes, González, Barrantes y Ortiz, 2011).

De este modo, no todo lo que se enseña responde a lo que estipula la política educativa sobre la interdisciplinariedad en las ciencias sociales; al respecto encontramos experiencias basadas en la historia oral, la historia reciente, la memoria colectiva y la historia social.

Así mismo, se encuentra el trabajo de rutas pedagógicas desarrollado por la RED, Universidad Nacional ${ }^{14}$, el cual, por demás, no se centra en recuperar experiencias y discursos que instalan la formación en competencias, sino en visibilizar la formación de una conciencia histórica, y dar cuenta de la relación entre el conocimiento disciplinar y la historia escolar.

Lo que sí queda de esta relación entre disciplinas y su enseñanza, para ampliar las tensiones y contrasentidos, es que

la enseñanza de las ciencias sociales está fuertemente influenciada por las disciplinas sociales desde una perspectiva regulativa y normativa, pues se indican los conceptos, nociones, estructuras o temáticas que se deben enseñar, sin reflexionar sobre las especificidades del saber escolar o de las condiciones institucionales en las cuales ocurren las prácticas de enseñanza y se constituye el código disciplinar ${ }^{15}$. (Cuesta, 1997, citado en Rodríguez y Acosta, 2007, p. 44).

14 En este trabajo se indaga por rutas pedagógicas que aportan a la enseñanza y estudio de la historia en la educación básica y media en cuatro instituciones educativas de Bogotá en grados $1^{\circ}$ a $9^{\circ}$. Allí se caracterizan los discursos y las prácticas pedagógicas referidas al conocimiento histórico y a su influencia en la construcción de conciencia histórica. Varios documentos evidencian la influencia de la historia social y la nueva historia en su enseñanza. Sobre la enseñanza de la historia, se puede consultar la propuesta de la SED sobre el campo histórico, desde una postura que reivindica la formación disciplinar de cara al diálogo interdisciplinar (Ocampo, 2007).

15 Se refieren al Estado del arte sobre la enseñanza y el aprendizaje de las ciencias sociales en los países de habla hispana (19002000). En este documento se contó con un acervo documental de 262 registros. 
O, como lo expresa Torres (2000), se trata de revisar las relaciones entre el saber histórico, más acá y más allá de la disciplina histórica, entendiendo que el saber histórico se refiere a lo que se produce y circula más allá de la disciplina histórica. De este modo, el saber histórico no es exclusivo de unos profesionales de la historia sino que forma parte de la cultura de todos los grupos sociales y se expresa en la memoria colectiva, en las tradiciones, la oralidad, las prácticas cotidianas y las representaciones que construyen sobre el pasado y el funcionamiento del mundo social.

Lo que está claro es que aunque hay relaciones con los campos disciplinares, el proceso de enseñar las ciencias sociales y la historia se constituye en un conocimiento pedagógico que genera desarrollos autónomos. Estos desarrollos mantienen el ideal herbartiano de que quien enseña debe saber de lo que enseña, no solo del cómo enseñarlo.

\section{Los asuntos comunes o de siempre}

En la mayoría de balances revisados hay un permanente cuestionamiento a lo que ha sido la enseñanza de la historia. Efectivamente, el problema no es que no se enseñe historia, sino qué historia se enseña. En efecto, en los balances se acude a la realización de caracterizaciones que definen la enseñanza tradicional de la historia, la revisión de manuales y textos escolares, y su función ideológica y moralizante. Veamos cada una de estas posturas.

Se entiende la enseñanza tradicional de la historia como aquella que aborda hechos históricos de manera aislada, al mantener una visión estática y fragmentaria de los contenidos, en la que se acude a la historia como memorización y repetición de lo ocurrido sin dar cabida a la interpretación y comprensión de problemas histórico-sociales. En esta concepción de enseñanza de la historia se mantienen temas repetitivos (Méndez, 2002), las periodizaciones de siempre, que van desde el periodo Precolombino, Conquista y Colonia, e Independencia (Medina, 2008; Aguilera y González, 2009), y los mismos héroes patrios.

En cuanto a los análisis que se han hecho de los manuales y textos escolares se encuentra la crítica a los idearios nacionales que se instauraron desde estos, lo cual responde a la formación de una identidad nacional moralizante, excluyente, racista y europeizante (Pinilla, 2003; Lenis, 2010, citado en González y Valencia, 2013). Al respecto, se le ha dado especial atención al Compendio de historia de Colombia de Henao y Arrubla (Pinilla, 2003; Arias, 2015; Álvarez, 2007). En otros casos la revisión de manuales y libros de texto se hace en función del análisis de la memoria oficial y las políticas de olvido que se instauran en estos. Así, se analiza la memoria oficial transmitida en el texto escolar de Henao y Arrubla, el cual se mantuvo en circulación hasta los años sesenta, por efecto de la articulación de dispositivos mnemotécnicos, de memorización y rememoración, que establecieron los principios de orden, verdad y valores hispánicos, desde los cuales se construyó la memoria dominante durante la primera mitad del siglo xx (Rodríguez, 2010). De igual manera, se revisa desde este texto escolar las políticas y prácticas de memoración (compendio de Henao y Arrubla), en las que se privilegia un relato de la patria basada en el orden, la civilización, el anticomunismo, y se excluye, a su vez, la visión de la movilización popular en el país del proyecto político de Gaitán, con lo cual se instaura una política de olvido sobre este momento histórico en la memoria escolar (Rodríguez, 2009). Esta mirada de la enseñanza de una historia moralizante y confesional se resume así:

El problema fundamental radica en que por varias generaciones el grueso de la población colombiana ha estado sometida a la enseñanza de una historia en esencia conservadora, predemocrática y contraria al fortalecimiento de la sociedad civil, que no ha posibilitado en el colombiano medio el desenvolvimiento de una conciencia crítica, social y moderna para hacer historia, fortalecer la democracia y lograr la paz y la armonía. (Equipo de Maestría, upN, 1999, p. 10).

\section{La didáctica de las ciencias sociales como campo}

Otro asunto común que ronda entre los investigadores del campo es la didáctica de las ciencias sociales (DCS). Este es uno de los aspectos más 
desarrollados, de allí que ya se hable de un campo como tal. A nivel general, se define la DCs desde las apuestas epistemológicas, en las que se abarcan las perspectivas, conceptos y métodos de investigación historiográfica que ofrecen claridad sobre desde dónde y cómo enseñar (Archila, 2004; Restrepo et al., 2000; Torres, 1995; Betancourt, 2008; Aguilera y González, 2008). Un segundo elemento es la alusión a los conceptos que estructuran la enseñanza de las ciencias sociales, y que en la mayoría de los casos referencia cómo se aprende el tiempo, el espacio y los grupos humanos (Aguilera y González, 2009), a la vez que se refieren a los conocimientos sobre cómo enseñarlas, teniendo en cuenta la edad de los sujetos a los que se dirige la propuesta de enseñanza. Ello indica que el campo de la DCs ${ }^{16}$ se constituye de referentes epistemológicos que, a su vez, son reconceptualizados en el proceso de enseñanza de las disciplinas sociales, de conceptos que en términos didácticos definen el abordaje metodológico de estas y del conocimiento sobre los sujetos del aprendizaje de las ciencias sociales.

De hecho, ya se arriesgan conceptualizaciones propias sobre el campo, al definirse la DCs "como el campo que se dedica a reflexionar con carácter riguroso sobre el proceso de enseñanza y de aprendizaje de las cuestiones o problemas de la formación cultural y social” (Quiroz y Díaz, 2011, p. 13). Si bien este es el aspecto más referenciado en los documentos revisados, también es en el que más cuestionamientos y avances encontramos. Por ejemplo, se mencionan los vacíos epistémicos en el campo, los cuales responden a que no hay acuerdos sobre los núcleos conceptuales o las categorías problémicas sobre las cuales se investiga desde la enseñanza de la historia y las ciencias sociales; es decir, hay una escasa claridad en los marcos de referencia en los que se apoya la investigación en el campo (Quiroz y Díaz, 2011). Este vacío se sustenta

16 Existen desarrollos y disputas en torno a la configuración de la DCs, ya que algunas posturas latinoamericanas, especialmente desde Argentina, la definen como una disciplina social, mientras otras perspectivas la entienden como un campo que se ocupa de la enseñanza de los saberes específicos, desde una perspectiva de las didácticas específicas. Se puede ampliar esta discusión en Aguilera y González (2008). en que, si bien se abordan categorías fuerza a la hora de pensar la enseñanza de las ciencias sociales (pensar históricamente, formar la conciencia crítica, pensar historiográficamente), estas no han tenido ni continuidad, ni reconocimiento en el ámbito nacional.

A ello se suma que no se ha logrado consolidar una comunidad epistémica, expresada en la conformación de redes, grupos de investigación, mayores niveles de producción, circulación y divulgación de estas, y en el escaso reconocimiento de los autores del campo en el ámbito nacional (Quiroz y Díaz, 2011; Rodríguez, 2013; Aguilera y González, 2009). Sin embargo, frente a este vacío se encuentran algunos avances al respecto, en términos de configurar redes, de lograr un mayor número de publicaciones y de la existencia de grupos y campos de investigación. En primer lugar, se cuenta con la Red Colombiana de Grupos de Investigación en Didáctica de las Ciencias Sociales, la cual fue creada en el 2010 y desde entonces ha convocado seis encuentros, en los cuales ha circulado buena parte de la producción en la DCS en Colombia. A ello se suma la reciente apertura del primer doctorado en Colombia en Didácticas Específicas de la Universidad Tecnológica de Pereira, en el que la DCs tiene un lugar asegurado.

En cuanto al número de publicaciones, se asiste a un incremento de la productividad académica. En el balance realizado por la profesora Sandra Rodríguez en el 2013 sobre la enseñanza de la historia en Colombia, se contó con un acervo documental de más de 200 registros, número significativo. De igual manera, se encuentra que dentro de las revistas de educación, ya hay números dedicados exclusivamente a la DCs, por ejemplo las revistas Educación y Pedagogía n. 34 (2001, Universidad de Antioquia); Pedagogía y Saberes n. ${ }^{\circ} 28$ (2008, UPN); Revista Unipliriversidad vol. 11, n. ${ }^{\circ} 2$ (2011, Universidad de Antioquia); y la Revista Estudios Sociales n. 52 (2015, Universidad de los Andes). En estas se alcanza a rastrear grupos y líneas de trabajo sobre la enseñanza de la historia y las ciencias sociales.

Por último, en la investigación liderada por Quiroz (2011) sobre las DCs en las universidades públicas de Colombia (Universidad Pedagógica Nacional, 
la Universidad de Pamplona, la Universidad de Córdoba, la Universidad de Tunja, la Universidad del Valle y la Universidad de Antioquia), encuentran que

de acuerdo con su denominación, solo tres grupos de investigación apuntan a la didáctica de las ciencias sociales: el grupo Sujetos y Nuevas Narrativas en la Investigación y Enseñanza de las Ciencias Sociales (Universidad Pedagógica Nacional, Bogotá), el grupo Com-prender - Didáctica de las Ciencias Sociales y Nuevas Ciudadanías (Universidad de Antioquia, Medellín) y el grupo Ciencias Sociales y Educación (Universidad del Valle del Cauca, Cali) ${ }^{17}$. (Quiroz y Díaz, 2011).

Este panorama ya motiva a hablar de un campo de investigación emergente, con posibilidad de consolidarse a futuro, $y$ atiende a los debates sobre el lugar de las disciplinas y las didácticas específicas.

\section{Encrucijadas de la memoria y la historia reciente}

Por último, se menciona con fuerza una línea de trabajo que se viene abriendo campo en la enseñanza de la historia y es la enseñanza de la historia reciente, y con ella los trabajos de la memoria en los que Elizabeth Jelin es un referente obligado. Desde esta perspectiva, Jelin (2002) propone algunas herramientas a la ahora de analizar los sentidos y la presencia de los pasados, en los que la memoria se aborda desde tres premisas:

Primero, entender las memorias como procesos subjetivos, anclados en experiencias y en marcas simbólicas y materiales. Segundo, reconocer a las memorias como objeto de disputas, conflictos y

17 En esta misma investigación se evidencia que son pocos los grupos de investigación que tienen líneas de trabajo en didáctica de las ciencias sociales. De las investigaciones en curso, seis se encuentran en este campo: "La enseñanza de las Ciencias Sociales" y "La enseñanza de las Ciencias Sociales en Colombia" (Universidad Distrital, Bogotá), "Pedagogía y didáctica para las Ciencias Geográficas y Ambientales" (Universidad Pedagógica y Tecnológica de Colombia, Tunja), “Didáctica de las Ciencias Sociales y nuevas ciudadanías" (Universidad de Antioquia, Medellín), "Epistemología y didáctica de las Ciencias Sociales" (Universidad Santiago de Cali, Cali) y "Enseñanza de la Geografía" (Universidad Pedagógica Nacional, Bogotá), lo que representa el $4,44 \%$ del total de líneas encontradas (Quiroz y Díaz, 2011). Actualmente estamos adelantando el estado del arte en la enseñanza de la historia y las ciencias sociales en la upN (2000-2014), que contribuye al campo. luchas $[\ldots]$ Tercero «historizar» las memorias, o sea, reconocer que existen cambios históricos en el sentido del pasado, así como en el lugar asignado a las memorias en diferentes sociedades, climas culturales, espacios de luchas políticas e ideológicas. (Jelin, 2002, p. 2).

Con estos sentidos se vienen realizando importantes abordajes del papel político de la memoria en torno a la lucha por la verdad, la no repetición, la reparación y el reconocimiento de las víctimas de hechos atroces. Es decir, esta postura confronta las historias y memorias oficiales en aras de reivindicar la memoria de las víctimas.

En el caso colombiano, Jiménez, Infante y Cortés (2012) realizan un estado del arte sobre los trabajos de la memoria en el contexto educativo ${ }^{18}$. En esta revisión se encuentra que los trabajos sobre la memoria en la escuela como

temática y dispositivo pedagógico de intervención, se institucionalizan en Colombia de manera paulatina en la primera década del siglo $\mathrm{xxI}$, producto del ejercicio mismo de la práctica docente y en momentos en que se da un proceso de "desmovilización" por parte de actores armados caracterizados como de derecha, lo que lleva a la sociedad colombiana a hablar, desde el año 2005, de una Ley de verdad, justicia y reparación de víctimas. (Jiménez, Infante y Cortés, 2012, p. 209).

Y aunque hace más de una década que se vienen adelantando investigaciones y experiencias concretas sobre la enseñanza de la historia reciente y la memoria, esta sigue siendo una demanda constante en

18 En este trabajo se incluyen abordajes desde la narrativa, la historia oral, la recuperación genealógica de la memoria activa del saber pedagógico, y la recuperación de la memoria pedagógica como perspectivas investigativas que confrontan las memorias oficiales. Para este caso solo retomamos lo reseñado en torno al uso político de la memoria en la historia reciente en torno a experiencias como la Red Interinstitucional de Memoria, del Instituto para la Paz, la Pedagógica y el Conflicto Urbano (Ipazud), de la Universidad Distrital, que desde el 2007 adelantan investigaciones y promueven publicaciones sobre el lugar de la memoria en el conflicto armado y en experiencias educativas del país. También se menciona el proyecto "Escuela, memoria y conflicto", Neira y Virginia (2012), así como los trabajos que las profesoras Sandra Patricia Rodríguez, Olga Marlene Sánchez y Martha Cecilia Herrera realizan desde la docencia universitaria al abordar los problemas de la enseñanza de la memoria en un país en guerra. 
términos de llevarla al currículo escolar (Rodríguez, 2013; González y Valencia, 2013).

Sin embargo, este abordaje de la memoria permite reflexionar sobre las memorias y los sujetos de un pasado más lejano, sus luchas por el reconocimiento que van más allá de la historia reciente, solo por citar un ejemplo. Aquíla pregunta que queda rondando es cómo desde los trabajos de la memoria en el campo pedagógico se articula la memoria larga y la memoria presente. Estas disputas por las memorias ponen de presente que debemos ser cuidadosos, pues cada memoria privilegia unos sujetos y hechos, es decir el ejercicio de la memoria selecciona sus propios olvidos. Parafraseando a Archila (2004), ¿cómo juega la historia reciente con los tiempos de larga, corta y mediana duración? ¿Qué es lo ejemplarizante en la memoria social que pueda abordarse en la memoria larga?

Sobre este aspecto, la misma Jelin (2012) advierte sobre la existencia de un efecto de saturación en los trabajos y prácticas sobre la memoria:

Es decir, si vamos a marcar todos los sitios donde tal o cual evento ocurrió, todos los horrores por los que hemos transitados, y todo se van a convertir en sitios de memoria, vamos a hacer más y más memoriales. ¿̇Hasta cuándo? Eso se convierte en una práctica habitual, ritualizada, pero segregada del resto de los procesos sociales. Es un peligro que debe quedar para la reflexión. Es algo que debemos imaginar y pensar, que los temas de memoria no solo giran alrededor de las violencias políticas del pasado reciente en Perú o en otros lados, sino que construyen sujetos colectivos donde los pasados se interpenetran, pasados de duraciones cortas con pasados de tiempos más largos. Quizás hay un déficit en nuestros trabajos sobre memoria que hace que nos quedemos en pasados recientes y no logremos incorporar ni un horizonte de futuro, ni un pasado más largo. (Jelin, entrevistada por Sandoval, 2012).

Así, la memoria, a secas, tendría la función política de controvertir las memorias oficiales del pasado lejano y del pasado reciente, y analizar los problemas que las convocan. Este aspecto de la memoria en la escuela se cruza con otras preocupaciones que han sido desconocidas en la memoria oficial y que también tienen una mirada de larga duración. Entre ellas está la historia económica, como posibilidad de comprender los problemas económicos y los modelos de acumulación en el mundo actual, en tanto desde estas comprensiones se puede entender problemas sociales (desplazamiento, pobreza, injusticia social, la minería, etc.) (Restrepo et al., 2000; González y Valencia, 2013). A su vez desde este aspecto se busca incluso incidir en la definición de los proyectos de vida y las visiones de futuro de los estudiantes.

Quizá porque históricamente la enseñanza y la didáctica de los saberes han sido conocimientos institucionalizados en el ámbito escolar, los procesos que no se han adelantado en contextos escolares no han sido rastreados. Por esto permanece la deuda con una sugerencia hecha por el profesor Alfonso Torres (1995), en la que alude de manera general a los esfuerzos de la educación popular por hacer historias alternativas, en las "que no se trata de cambiar de héroes y contenidos con la misma perspectiva de enseñanza, sino de hacer de la historia otra cosa". Estos esfuerzos permanecen aislados y, por tanto, aún no se tienen perspectivas sobre el abordaje de la enseñanza de la historia con poblaciones populares, indígenas, adultas, marginales, en los movimientos sociales e incluso en las innovaciones educativas. Este es un aspecto nodal pues muchas de estas experiencias desde apuestas emancipadoras pueden aportar a la enseñanza de la historia en la escuela.

Efectivamente los contrasentidos, las tensiones y los retos enunciados son cuestionamientos mismos a la estructura escolar cimentada en la fragmentación de la vida, de los asuntos del pensamiento y del conocimiento, distribuidos en horas de 45 minutos, en cambios de clase, en la división de asignaturas, que no permiten entender los problemas sociales que encuentran comprensiones históricas en el presente y que avizoran sueños colectivos. 


\section{Referencias}

Aguilera M., A. y González T., I. (2009). Didáctica de las ciencias sociales para la educación infantil. Análisis, propuestas y estado de la cuestión. Bogotá: UPN.

Álvarez G., A. (2007). Ciencias sociales, escuela y Nación. Colombia 1930-1960 (tesis inédita de doctorado). Universidad Nacional de Educación a Distancia (UNED), Madrid, España.

Equipo de Maestría en Enseñanza de la Historia del Departamento de Ciencias Sociales, Universidad Pedagógica Nacional (1999). La enseñanza de las ciencias sociales en Colombia. Balance y perspectivas. Revista Educación y Cultura, (47), 5-10.

González V., G. y Valencia, C. H. (2013). La didáctica de las ciencias sociales en Colombia: planteamientos teóricos y estado de la cuestión de la investigación. En J. Pagés y A. Santisteban (eds.), Una mirada al pasado y un proyecto de futuro. Investigación e innovación en didáctica de las ciencias sociales. Vol. 1. Barcelona: Asociación Universitaria de Profesorado de Didáctica de las Ciencias Sociales, Universidad Autónoma de Barcelona.

Guerrero G., C. (2011). La incidencia de las reformas educativas en la enseñanza de la historia en Colombia, 1973-2007 (tesis inédita de maestría). Universidad Nacional de Colombia, Bogotá, Colombia.

Jiménez, A., Infante R. y Cortés, R. A. (2012). Escuela, memoria y conflicto en Colombia. Un ejercicio del estado del arte de la temática. Revista Colombiana de Educación, (62), pp. 287-314.

Ley 115 de 1995 (8 de febrero), por la cual se expide la Ley General de Educación.

Medina Pineda, M. (2008). La enseñanza de la historia en los países miembros de la Comunidad Andina de Naciones. Informe Colombia. Bogotá: Convenio Andrés Bello, Universidad Andina Simón Bolívar, Secretaria General de la Comunidad Andina.

Méndez Montenegro, I. (2002). Hacia una didáctica de la historia: Análisis de las propuestas de enseñanza de la historia que se publicaron durante la última década del siglo $\mathrm{XX}$ (tesis de inédita de pregrado). Universidad Pedagógica Nacional, Bogotá, Colombia.

Neira U., Virginia, A. (2012). Memoria, conflicto y escuela: voces y experiencias de maestros y maestras en Bogotá. IDEP. Bogotá.

Ortiz, J. G. (Icfes), Ayala Diago, C. A. (U. Nacional), Chaparro, J. (U. Nacional), Sarmiento Lozano, J.
(U. Nacional), Restrepo, G. (U. Nacional) (2007). Fundamentación conceptual área de ciencias sociales. Bogotá: Icfes.

Quiroz Posada, R. E. y Díaz Monsalve A. E. (2011). La investigación en el campo de la didáctica de las ciencias sociales y su dinámica de articulación en un grupo de universidades públicas en Colombia. Revista Unipluriversidad, 11(2), 1-16.

Restrepo, G., Ramos Reyes, J. A. y Sarmiento Lozano, J. L. (2000). Hacia unos fundamentos de la enseñanzay el aprendizaje de las ciencias sociales en la educación media. Bogotá: Universidad Nacional de Colombia.

Rodríguez, S. P. (2014). Enseñanza y aprendizaje de la historia en Colombia 1990-2011, pp. 109-154. En S. Plá y J. Pagès (comps), La investigación en la enseñanza de la historia en América Latina. México: Bonilla Artigas editores/UPN.

Rodríguez Ávila, S. y Acosta, W. (2007). Estado del arte sobre la enseñanza y el aprendizaje de las ciencias sociales: Producción académica en los países de habla hispana 1990-2000. Informe Final de Investigación. Bogotá: CIUP-UPN.

Torres Carrillo, A. (1995). La enseñanza de la historia en Colombia. Notas sobre el estado de las búsquedas actuales. Revista Conjeturas, (1), 37-70.

\section{Referencias complementarias}

Aguilera Morales, A. y González T., M. I. (2008). La didáctica de las ciencias sociales y/o la didáctica como ciencia social. Revista Pedagogía y Saberes, (28), pp. 107-114.

Álvarez Gallego, A. (2013). Las ciencias sociales en Colombia: Genealogías pedagógicas. Bogotá: IDEP.

Archila, M. (2004). El historiador y la enseñanza de la historia. En J. G. Rodríguez, Rutas pedagógicas de la historia en la educación básica de Bogotá. $1^{\text {a }}$ ed. Bogotá: RED- Universidad Nacional de Colombia.

Arias G., D. (2015). La enseñanza de las ciencias sociales en Colombia: Lugar de las disciplinas y disputa por la hegemonía de un saber. Revista de Estudios Sociales, (52), pp. 134-146.

De Sousa Santos, B. (2009). Hacia una sociología de las ausencias. En Una epistemología del Sur. Argentina: Clacso/Siglo Xxi.

Betancourt, D. (2008). Enseñanza de la historia a tres niveles. Bogotá: Magisterio. 
Castro Bueno, F. (2004). Los proyectos de investigación histórica escolar. Revista Educación y Cultura, n. ${ }^{\circ}$ 65, 68-75.

Cortés, A., González, M., Barrantes R. y Ortiz M. C. (2011). Las rutas pedagógicas en la enseñanza de la historia. Bogotá: IDEP-OEI.

Martinic, S. (2001). Conflictos políticos e interacciones comunicativas en las reformas educativas en América Latina. Revista OEI, (27), pp. 17-34.

Pinilla, A. V. (2003). El compendio de historia de Colombia de Henao y Arrubla y la difusión del imaginario nacional a comienzos del siglo xx. Revista Colombiana de Educación, 45(90), 90-117.

Rodríguez A., S. (2013). Aproximación a la formación de profesores en ciencias sociales y en historia en Colombia: Elementos para el debate. En Reseñas de la enseñanza de la historia (pp.29-56). Argentina: APEHUN.

Rodríguez A., S. (2010). Construcción de la memoria oficial en el Centenario de la Independencia: El Compendio de Historia de Colombia de Henao y Arrubla. Revista Folios, (32), 23-41.

Rodríguez A., S. (2009). El 9 de Abril en las políticas de la memoria oficial: El texto escolar como dispositivo de olvido. En C. A. Ayala Diago, O. J. Casallas y H. Cruz Villalobos, Mataron a Gaitán: 60 años (pp. 135-154). Bogotá: Facultad de Ciencias Humanas, Universidad Nacional de Colombia.

Sandoval, P. (2012). Entrevista a Elizabeth Jelin. Revista Argumentos, 5. Recuperado de: http://revistaargu- mentos.iep.org.pe/articulos/entrevista-a-elizabeth-jelin/

Silva Briceño, O. (2013). Balance historiográfico sobre la enseñanza de las ciencias sociales en Colombia. En Memorias del Coloquio Internacional sobre Educación Pedagogía y Didáctica: Problemas Contemporáneos Bogotá: Universidad Distrital.

Thompson, Edward (1987). Miseria de la teoría. Barcelona: Crítica.

Torres Carrillo, A. (2000). Comprensión crítica, nuevas narrativas y enseñanza de la historia. Educación Hoy, (144), 73-92.

Vega, R. (1998). La historia oral, enseñanza de la historia $y$ conocimiento. Bogotá: Anthropos.

Vega Cantor, R. y Castaño Támara, R. (1999). Déjenos hablar. Profesores y estudiantes tejen historias en el espacio escolar. Bogotá: IDEP.

Velasco, G. y Castaño, R. (2006). Aprender historia haciendo historia. Bogotá: Universidad Distrital Francisco José de Caldas.

Wallerstein, I., Calestous, J., Fox Keller, E., Kocka, J., Lecourt, D., Mudimbe, V. Y., Mushakoji, K., Prigogine, I., Taylor, P. J., Trouillot, M. R. y Lee, R. (1996). Abrir las ciencias sociales. México: Siglo xxi/Centro de Investigaciones Interdisciplinarias en Ciencias y Humanidades, UNAM.

Wallerstein, I. (1997). La historia de las ciencias sociales. México: UNAM.

Para citar este artículo

Aguilera, A. (2017). La enseñanza de la historia y las ciencias sociales hoy: contrasentidos y posibilidades. Folios, (46), 15-27. 